\title{
A Comprehensive Overview of Digital Signal Processing Methods for Voltage Disturbance Detection and Analysis in Modern Distribution Grids with Distributed Generation
}

\author{
Aleksandar M. Stanisavljević, Vladimir A. Katić, \\ Boris P. Dumnić, Bane P. Popadić
}

University of Novi Sad, Faculty of Technical Sciences, Trg Dositeja Obradovića 6, 21000 Novi Sad, Serbia, acas@uns.ac.rs, katav@uns.ac.rs, dumnic@uns.ac.rs, banep@uns.ac.rs

Abstract: The rapid trends towards smart grids and implementation of distributed generation $(D G)$ and renewable energy sources bring new challenges in power quality domain. Modern distribution grids have a higher amount of voltage disturbances due to $D G$ sower converters, nonlinear loads and system faults. The on-going research on development of new, faster and more reliable techniques for detection and analysis of voltage variations in order to prevent malfunction of equipment or to support gird and enhanced its operation, is at present very important topic. The paper presents a comprehensive overview of voltage disturbances detection and analysis methods, which use different digital signal processing techniques for use in modern distribution grids. Comprehensive, critical literature review encompassed wide range of methods, from standard, well-known ones over digital signal processing (DSP) ones to the advanced, hybrid methods. Simulation and laboratory evaluation of methods applied as part of gridtie inverter control is presented. Advantages and disadvantages are underlined and critical evaluation of selected methods is presented. The main criteria for evaluation of methods are the speed of detection, a reliability of methods, analysis capability and computational complexity (i.e. cost of application).

Keywords: Power quality disturbances; fault analysis; artificial intelligence; signal processing; smart grids

\section{Introduction}

Modern concept of smart grid implies multilayer structure around power system with wide application of digital technologies and encompasses integration of energy network with digital communication network, wide-area measurements, powerful computer data processing, management and large data bases. In energy layer, it enables two way energy flows due to connections of distributed 
generation (DG), renewable energy sources (RES), electric vehicles with energy recovery feature, fast energy storage, high efficient and sophistically controlled industrial and domestic loads and other devices making distribution network active one. Most of them are connected to the grid with some type of power electronics (PE) systems. It could be grid-tied inverter (DC/AC converter), as in case of PV systems, or grid-tied rectifier (AC/DC converter) in cases of industrial or domestic loads, or their combination, AC/DC/AC (back-to-back) converter, as in cases of some types of wind generators or some other PE converter.

All these PE systems are sensitive to voltage disturbance (VD) in the grid. The major disturbances are large power variation, either on load side or on generation side (in case of renewable generation) leading to voltage variation and unbalance. Another set of voltage disturbances result from different type of faults (shortcircuits) resulting in voltage interruptions, voltage dips (sags), voltage swells or other. These disturbances affect proper operation of different loads, especially sensitive ones, cause load tripping, overheating and might produce significant economic and production losses [1]. The generation units are affected, also, especially in cases of voltage dips. On the other hand, power electronic devices having non-linear characteristics induce additional distortion on voltage waveform (harmonics, flickers, etc.). There are also other sources of VDs, like overvoltage due to lightning strikes, impulses due to capacitor bank switching, etc.

In this paper, focus will be on VD, especially on voltage dips and their interaction with DGs. In such cases PE devices are subject to high over current stresses, errors in synchronization circuits (PLL), increase of current distortion and other effects, which may result in their tripping. However, according to recent grid codes the PE based generation units need to stay connected to the grid during the voltage dips (for a defined period of time) and support the grid by supplying some amount of reactive and active power or only reactive power, depending on the voltage dip depth $[2,3]$.

The first step in reducing the effects of VDs, especially of voltage dips, is fast and reliable detection. The control system (as a part of PV system connected to the LV or MV grid) should switch from the normal operation mode to grid fault operation mode as soon as possible[4]. In that case, behaviour of the whole control system of the grid-tie converter or similar device may be swiftly adopted to low voltage ride-through (LVRT) requirements. Also, there are different applications of voltage dips detection and analysis (VDDaA) methods in Dynamic Voltage Restorers (DVRs) [1, 5-7], Series and Shunt controllers based on voltage-source converters, Unified Power Quality Conditioning Systems (UPQCSs) [1, 6, 8, 9], microprocessor relay protection [10], DGs control algorithms [3, 11-13], PQ monitoring algorithms $[14,15]$ and FACTS. For all these systems, it is desirable that VD is detected with the shortest delay that is achievable.

In modern power systems a large number of voltage disturbances data, which may be recorded makes analysis very complex [16]. Many researchers have applied some type of the digital signal processing (DSP) based methods [17, 18] for 
VDDaA. They are using a large collected scientific experience from other fields, like telerobotics [19], numeric estimation [20] or nanostructure analysis [21] to name a few. The existing paper reviews [14, 22-29] present mainly wide-range overview of the technical literature based on comparison of results given in these papers. They are obtained in different conditions and for different PE systems. From these references, it can be concluded that proposed algorithms for different voltage dips detection and analysis (VDDaA) are tested by computer simulations, only. Also, it can be observed that the main advantage of new methods is in their ability to detect and analyse multiple disturbances and to successfully classify them even in noisy conditions [22, 25, 30, 31].

In this paper focus is on application of VDDaA methods in distribution grids, with special emphasis on characteristics that are important for applications in such conditions. The paper's aim is to present a comprehensive and critical literature overview for VDDaA methods. Detailed classification of these methods is given. Based on reviewed literature, comparison of selected VDDaA methods is presented. Also, for compared methods advantages and disadvantages are highlighted. The comparison is made according to the three main criteria: speed of detection, analysis capability and complexity and cost of implementation.

The main contribution of the paper is that comparison and evaluation of VDDaA methods are done under the same conditions and performed by experimental testing in laboratory using both grid emulator generated voltage dips and voltage dips measured in real grid. The comparison is done in the case of application of all these methods for control of a grid-tied inverter using three mentioned criteria and by evaluating each result with specific unique grade (from 1 to 10). In this case the optimal method may be selected with more reliability than in previous reported researches.

The practical value of this overview is that it may be a relevant source for insight in potential and features of a broad spectrum of VDDaA methods. Also, the best ones can be used as part of grid-connected converters control, in LVRT support algorithms, PQ monitoring devices or for other applications. By using of the selected optimal method significant improvement in the control algorithm of these PE devices is possible, i.e. control engineers will have possibility to achieve better performances and capabilities of the control systems.

The paper is organized as follows. Theoretical background is given in the second section and contains brief description of the PQ standards and basics on voltage disturbance and analysis algorithms. In the third section a comprehensive critical overview of VDDaA methods with classification is presented. In the fourth section, the results of comparison of previously reported methods and the ones achieved by laboratory testing using real measurement data and grid emulation are presented and described. The conclusions, future scope, acknowledgements and references are given in final part of the paper. 


\section{Theoretical Background}

This section describes a theoretical background on important power quality standards and gives details of the VDDaA algorithm.

\subsection{Voltage Dip}

Voltage signal should be less than $90 \%$ of the RMS nominal voltage value to consider it a voltage dip and perform detection. The common detection method is the RMS. This method is standard one, and for many years it has been used in practice [1]. Voltage dips can be classified in different ways, for example, using voltage amplitude and phase angle variation, $\mathrm{ABC}$ classification (7 types of dips) [1], or using amplitude time change and measuring duration of dips.

\subsection{Power Quality Standards}

Harmonics in power systems attracted a lot of attention and large effort is made in order to achieve accurate estimation and reliable mitigation of them. Many standards, guidelines and recommendations are published, including IEEE 5192014, EN 50160 and several IEC 61000 standards (6100-4-30) [32].

Also, other VDs are addressed in several other IEEE standards. In the IEEE 11592009 the classification and definition of VD are presented. According to it voltage dips are defined as a decrease of 0.1-0.9 p.u. in the voltage magnitude at system frequency with the duration of half cycle to 1 min [33]. The IEEE Std. 1564-2014 identifies, describes and defines appropriate voltage dip indices, as well as characteristics of electrical power systems [34].

\subsection{Voltage Disturbance Detection and Analysis}

Normal duration for voltage dip detection that a standard VD algorithm requires is 1 to 2 grid cycles. Such a reaction time may not be always appropriate, as modern grids have new types of PE equipment and grid requirements are upgraded.

VD analysis is a complex task which can be divided in several stages. The first stage of VD analysis is measurement. Depending on the application, type of device and equipment, measurement usually includes some sort of transformers. Measurement can also include sampling, analogue anti-aliasing filtering, downsampling, or other signal preparation steps. The next step, after measurement, is transforming voltage signal values from analogue to digital (A/D conversion). This paper assumes that $\mathrm{A} / \mathrm{D}$ conversion is done without any errors and with sufficient sampling rate according to the Niquist-Shennons theorem [32]. A simplified algorithm of typical VDDaA scheme (based on algorithm described in [32]) is shown in Fig. 1. Digital waveform data are then pre-processed with a transformation or feature extraction, as the voltage waveform cannot be directly 
used to detect VDs (and any other PQ disturbance) [32]. Also, it is not suitable for voltage magnitude analysis. For example, for voltage dips detection the one-cycle RMS voltage should be compared with a $90 \%$ value of magnitude every half cycle, according to IEEE 1564-2014 [34] and IEC 61000-4-30 [35]. Once the disturbance is detected, this information can be used in devices to aid LVRT, or in inverter control for choosing proper power profiles (if it is recognized as voltage dip) [4]. After detection, the so-called single-event indices (also known as singleevent characteristics) which typically include duration and some kinds of magnitude are obtained. Besides these, actual analysis differs for different types of events, and signal can be further analysed and other indices can be calculated and stored or further processed. Some of these indices are depth of a dip, phase shift, voltage dip energy (Evs), voltage dip severity (Se), system index (a parameter indicating the voltage or current quality), harmonics, type of fault, estimated distance of fault, harmonics, etc. These data can be used for diagnostics, for calculating additional fault parameters and causes of disturbance, improvement of control, in PQ classification algorithms, etc.

\section{Overview and Classification of Voltage Disturbances Detection and Analysis Methods}

A large number of papers that present new methods and algorithms for detection and analysis of voltage disturbances are published in the last two decades. Mostly, they use some type of the digital signal processing (DSP) algorithm to extract features and further analyse them, to obtain detection or classification of the disturbances, to estimate there's a characteristic, to calculate distance of the fault, etc. General classification of the voltage disturbance detection and analysis methods is presented in Fig. 2. It can be seen that the DSP methods for VDDaA can be divided in the three large categories: Standard DSP methods, DSP based methods and DSP and AI based methods.

Both voltage dips and voltage variations use the RMS of voltage as their basic measurement quantity [32, 34, 35]. Because of that, the RMS method is the most commonly used method for detection and segmentation. According to IEC 610004-30 [35] for the detection of voltage dips, the one-cycle RMS voltage value is

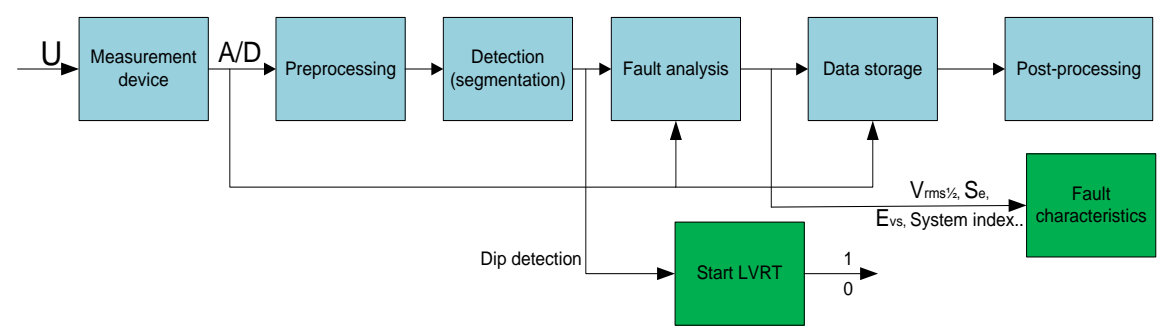

Figure 1

A general scheme of voltage dips detection and analysis methods 
compared with a threshold every half cycle, also, in IEEE Std. 1564-2014 [34] for voltage dip characteristic voltage, depth of dip, etc. In addition, several different variations of the RMS method exist and they can be characterized as advanced RMS calculation methods. Because RMS methods are well known, they will be only briefly addressed.

The second group of DSP VDDaA methods is DSP methods based on transforms (or just DSP based methods). Algorithms in this group use mathematical transforms (usually harmonic estimation) to obtain voltage disturbance indices in time, frequency, or other domain. Based on transformed signals, they detect and further analyse disturbances. This is probably the largest group, which is further developed in several different directions.

The third group, most up-to-date, covers methods that utilize some form of artificial intelligence (AI). The AI is used in order to improve performances of detection and analysis. Comparing a feature of voltage, e.g. RMS with the threshold (0.9 p.u.) is replaced with complex pattern recognition and learning models. Usually, some form of neural networks (NN) or Fuzzy logic (FL) is used to improve detection and analysis of disturbances. For pre-processing or segmentation these methods use some of the DSP methods, e.g. Wavelet transform (WT), FFT, Hilbert-Huang transform (HHT), Short Time Fourier transform (STFT), S-transform (ST), etc. These methods are not the topics of this paper, because they are still in developing and methods are not common in applications that are addressed.

\subsection{Standard DSP Methods}

The voltage waveform cannot be directly used to detect or classify events. Because of that, simple and the most common methods are based on the direct extraction of the voltage magnitude RMS from the voltage waveform. Also, very frequent approach is to calculate fundamental-voltage magnitude sequences (the approximated RMS) and to detect and analyse disturbances on the basis of that.

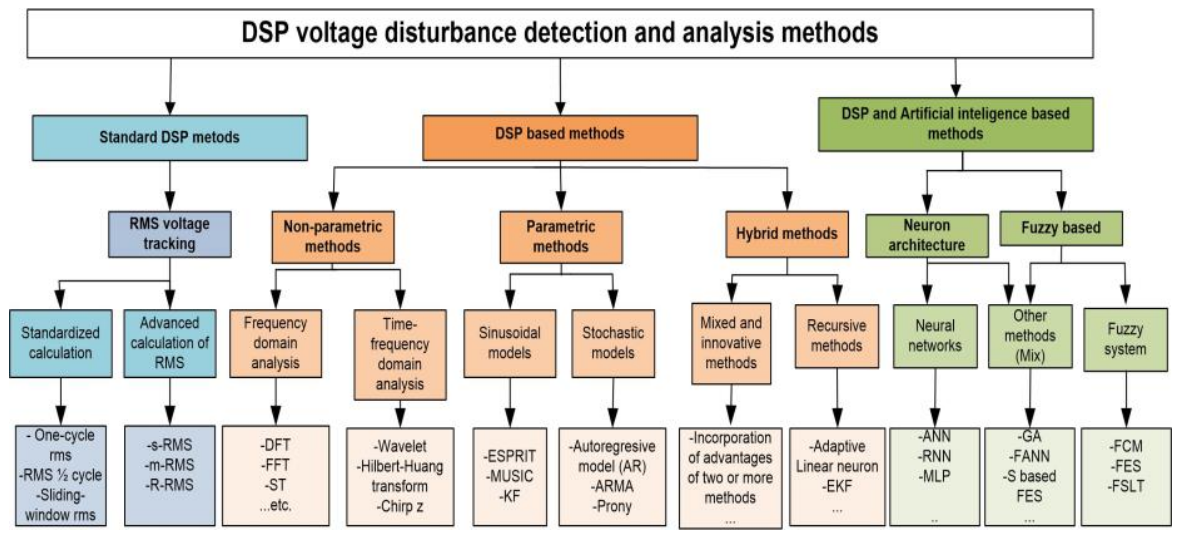

Figure 2

Classification of voltage disturbance detection and analysis methods 
An important parameter for the RMS is block (buffer) size of a data sequence that is analysed. RMS obtained from using a half-cycle window has a higher time resolution, but with more fluctuation compared with RMS obtained from a onecycle window [32]. Voltage RMS magnitude is usually obtained from discrete signal using (1).

$V_{r m s}=\sqrt{1 / 2 * \sum_{n=1}^{N} v_{i}^{2}}$

where $N$ is a number of samples (buffer size), $n$ is the $n^{\text {th }}$ sample of the data and $v_{i}$ is digital voltage signal.

In IEEE [34] and IEC [35] standards, $\mathrm{V}_{\mathrm{rms}} 1 / 2$ is defined as a value of RMS voltage measured during one cycle and results are updated each half cycle (RMS 1/2 cycle). In [36] different ways in which RMS can be calculated are presented, using fix window of different durations (s-RMS), moving average technique (m-RMS) or infinite impulse response (recursive moving average, r-RMS). If the RMS is continuously calculated over a windowed signal, using past samples from an input, it is called a moving average finite impulse response (FIR) filtering, and it is abbreviated as s-RMS [36].

Beside delay in detection, limitation of estimation of magnitude and duration (especially for short duration faults), as well as inability to calculate phase-angle information nor the point-on-wave when fault starts are drawbacks of these methods [37].

\subsection{DSP-based Methods}

DSP based methods for VDDaA include methods that use various types of transformations and can be further divided into three sub-categories as nonparametric methods (NPM), parametric methods (PM) and hybrid methods (HM).

\subsubsection{Non-Parametric Methods}

The NPMs have low computational complexity. They calculate harmonics with algorithms that are applied directly on discretized voltage waveform [38]. Also, they are well-known, easy to use and implementation costs of these methods are low.

In literature, two subgroups of the NPMs can be found: Frequency domain analysis and Time-frequency domain analysis [38]. Transformation from time domain to the frequency domain is usually done with FFT. The FFT is a way of calculation of the DFT that can be defined as in (2).

$H(m)=\sum_{n=0}^{N-1} x(n) * e^{-j(2 \pi / N) k n}$

where $H(m)$ is calculated harmonic, $n$ is the $n^{\text {th }}$ sample of the data, $m$ is frequency index and $N$ is number of samples (buffer size). 
However, FFT has many known problems: leakage effect, sensitivity to frequency deviation, etc. [32, 39]. Many researchers proposed new solutions in order to improve FFT and to solve these well-known problems. Newly proposed algorithms try to improve FFT using synchronization [40, 41], windowing [42, 43], interpolation [44, 45] or using different sampling techniques [46], etc. Besides these algorithms, new NPMs emerged. Many of them are developed for PQ event detection and analysis and become widely used, like WT [47, 48] and HHT [39, 49]. Also, advanced successors of the FT and FFT, like S-transform or STFT, show very good results in different applications (detection of faults in modern grids [50] or in power quality analysis [51, 52]).

The WT is one of the most commonly used methods for harmonic analysis in PQ associated applications. The WT estimates a local representation of signal in a time domain and in a frequency domain, and this is usually consider as timefrequency representation. The discrete WT can be calculated as shown in (3).

$\mathrm{F}(i, j)=L^{-j / 2} * \sum_{i=0}^{N} \mathrm{f}(\mathrm{n}) * \omega *\left(\frac{n-i}{L^{j}}\right)$

where $v_{i}$ is digital voltage signal, $F_{i j}$ is matrix that consists of decomposed $v_{i}$ values, $j$ is the level of the decomposition, $i$ is band index, $L$ is dilatation translation parameter (for Dyadric wavelets it is equal to 2), $N$ is number of samples, $\omega$ is complex conjugate and $n$ is $n$th sample of the data [32]. It shows very good results as tool for analysing fast-changing signals, like VDDaA [53]. Mostly, the highest frequency band is used for detection of voltage disturbances [32]. In [54] method for VDDaA, with WT used as a tool for detection and extraction of useful information from disturbance is presented. Probabilistic NN (PNN) is used for detection of patterns and classification. A main disadvantage of wavelets is that the centre frequencies of the sub band filters are difficult to be set in the harmonic frequencies, making them less attractive to harmonic-related disturbance analysis [32]. Also, detection using wavelets is prone to noise and signal deterioration [55-57].

S-transform (ST) is modified version of WT that is well-known, mainly for application in PQ analysis and classification algorithms [58]. In [59] comparative study for wavelet and ST for PQ disturbance detection, analysis and is landing detection is presented. It is concluded that $S$-transform is better than wavelet for detection and localization of PQ events based on simulations and experimental results. Mathematical model of ST (continuous integration formulation) can be written as in (4):

$$
\mathrm{S}(\tau, j)=\int_{-\infty}^{\infty} \mathrm{x}(\mathrm{t}) \mathrm{w}(\mathrm{t}-\tau, j) d t
$$

where $\mathrm{w}[\mathrm{t}-\tau, j]$ is a scaled replica of the fundamental mother wavelet, as defined for WT, $t$ in this case is dilation that determines the width of the wavelet and resolution of transformation. Further, if for multiplication is used function $\mathrm{S}$ :

$$
S=e^{i 2 \pi f t}
$$


And for mother wavelet is used function w:

$$
w(t, f)=\frac{f}{\sqrt{2 \pi}} e^{\frac{-t^{2} f^{2}}{2}} e^{-2 \pi i f t}
$$

The final form of ST combined (4-6) can be written as [60]:

$$
\mathrm{S}(\tau, f)=\int_{-\infty}^{\infty} \mathrm{x}(\mathrm{t}) \frac{f}{\sqrt{2 \pi}} e^{\frac{-t^{2} f^{2}}{2}} e^{-2 \pi i f t} d t
$$

In [61] the analysis of voltage disturbance with WT and STFT methods is discussed. From studies and examples presented in this paper, advantages and disadvantages of WT and STFT are described. Both methods are able to detect the transient of disturbance. STFT is better for time-frequency analysis of disturbances, while WT presents better results for detecting events. Both methods are very similar and they showed similar results. STFT, as alternative to FFT, differs from FFT because it uses a window function $\mathrm{w}[\mathrm{n}-\mathrm{m}]$, and this window translate in time by $\mathrm{m}$ samples. STFT can be defined as a sum, as presented in (8).

$$
\mathrm{FS}_{\mathrm{i}, \mathrm{j}}=\sum_{n=0}^{N} \mathrm{x}[\mathrm{n}] e^{-\frac{2 j \pi k n}{N}} \mathrm{w}[\mathrm{n}-\mathrm{m}]
$$

where $w[n-m]$ is window function and $x[n]$ is $n^{\text {th }}$ digital sample of voltage signal.

Research which also compares PQ analysis capabilities of WT and STFT is presented in [52]. Conclusion of this research is similar to the conclusion presented in [61], i.e. STFT is more suitable for disturbance signal analysis, while WT obtained better results for detection of disturbances. In [62], different PQ VDDaA methods are presented and compared. Between RMS, STFT and high pass filter, STFT showed the best results. In [37], a comparative study of RMS, DFT, EKF and WT for detection and analysis of voltage disturbances is presented. In this paper, it is concluded that STFT and RMS methods in all tested cases have delay in detection, EKF shows good results and WT shows the best results in the detection and analysis. However, WT must be used with other method in order to differentiate voltage disturbances from frequency disturbances. In [63] comparison of KF, WT and FFT for voltage dip parameters estimation is addressed. The methods are tested with different signals, including signal with noise, phase angle jump, etc. In this paper, it is concluded that WT is prone to noise and other disturbances with higher frequency components, and that KF and FFT performances are acceptable and satisfy mitigation requirements. Also, it is concluded that the RMS shows the worst results in comparison. In [64] two methods for voltage dip detection are tested as part of grid-tie inverter system. Reduced FFT (RFFT) method shows better results in comparison with FFT, both in speed of detection and in complexity.

HHT is signal analysis method, which consists of two-part transformation, the empirical mode decomposition (EMD) and Hilbert transformation. The HHT of the signal in time domain calculates also real valued time domain signal $\overline{x(t)}$. This 
two values can form analytical signal: $z(t)=x(t)+j \overline{x(t)}$, where $\mathrm{x}(\mathrm{t})$ is original signal. Transformation can be written as [22]:

$$
\overline{x(t)}=\int_{-\infty}^{\infty} \frac{x(\tau)}{\pi(t-\tau)}
$$

The amplitude signal and instantaneous phase angle $\theta(t)$ and frequency $f_{0}$ can be written as (10-12):

$$
\begin{aligned}
& A(t)=\left[x^{2}(t)+\overline{x^{2}(t)}\right]^{1 / 2} \\
& \theta(t)=\frac{1}{\tan (\overline{x(t)} / x(t))} \\
& f_{0}=\frac{1}{2 \pi t} \frac{1}{\tan (\overline{x(t)} / x(t))}
\end{aligned}
$$

HHT is often used as part of algorithms for PQ detection and classification. In [65] application of HHT in wind power systems for voltage dips detection is presented. It is shown that HHT can successfully detect a dip with good detection times, very accurately, but only voltage dips are examined, in simulations, and further examination of this method as stand-alone is needed. In [66] method based on HHT and Symbolic Aggregate appro Ximation (SAX) is proposed for analysis and identification of sudden changes in waveform. The method is tested for general sudden changes and non-stationary signals, to identify frequency amplitude and phase angle. Tests for any type of real PQ disturbance for detection, identification or analysis are not performed in the paper. In [67] HHT method is used for detection, analysis and classification, only with addition of fuzzy rules in classification part of method. Both detection and analysis of single and multiple disturbances are tested. It is stated that HHT can extract from disturbance signal instantaneous amplitude, frequency and phase. Also, many features of disturbances can be calculated from this data set.

From presented literature review, it can be concluded that results of specific methods depend on their application. However, some methods present better results in most of the applications, while others always underperform. Methods based on WT, FFT, HHT and ST in most cases show at least good results, while RMS usually shows the worst results. HHT and ST are mainly used as part of PQ classification algorithms.

\subsubsection{Parametric Methods}

PMs are the second most important group of DSP based methods. This class of methods use model of signal to perform analysis. Appropriate model is chosen based on knowledge about signals properties. If the model has good matching with the signal, this type of method can achieve high accuracy [32]. Otherwise, if signal is not properly modelled, PM methods can induce significant error. 
In [68] AutoRegressive model (AR) is applied for VDDaA. In this paper, it is shown that AR models can be used for detection transitions and potential for tracking time behaviour of dominant frequency, and that this method can be used for event analysing, but that further studies are needed. In [69] detection of voltage disturbances in noisy signals is addressed using AR model in combination with sequential generalized local likelihood ratio detector. In presented simulations, superior performance of proposed method is observed.

In [70] performances of Adaptive Linear Neurone (ADALINE) based method is compared with RMS, WT and HPF for detection of voltage dips. ADALINE is an adaptive filter that is usually used for extraction of waveform features from signals and for reducing noise. In this paper, it is concluded that problem of ADALINE method, as well as AR and ARMA methods, is classification of disturbances. Also, problem for these methods is determination of threshold value that is used for detection. For WT, it is concluded that WT is suitable for the detection of PQ disturbances, but analysis of disturbance is sensitive to noise.

Kalman filter is a method that shows good results in voltage disturbance detection and analysis. This method has good accuracy in amplitude estimation, phase and frequency estimation for application in analysis of disturbance [71]. Method that uses three KF for detection of voltage events and to estimate single-event characteristics is presented in [72]. Results of method using real-grid measurements, applied in real-time environment shows that method is suitable for detection of voltage disturbances, with much faster detection in comparison with RMS $1 / 2$. Results for precision and reliability of method are not presented.

The Estimation of signal parameters via rotation invariance technique (ESPRIT) and the Multiple Signal Classification (MUSIC) method can be applied for stationary signals analysis [32]. These methods can be further upgraded to work with sliding-window processing methods or as block-based processing methods and can be used to analyse non-stationary signal, but this requires further research.

It can be concluded that PM methods are suitable mainly for analysis of disturbances. Also, these methods can be good choice for offline processing where a delay is required or for improving reliability of classification [38].

\subsubsection{Hybrid Methods}

HM are mainly methods that cannot be classified as previously addressed groups and do not have implemented some of the AI algorithms. Current classification of DSP (or just signal processing) methods known in literature [32, 38] is further upgraded in [73]. HM can be divided in two sub-groups: Mixed and innovative methods and Recursive methods.

A new method for detection and analysis of VDs which is a combination of WT and sliding-window is presented in [74]. WT is used for detection and good results, even for noisy signals, are obtained. However, in this paper, accuracy and 
reliability of method, as well as the exact time delay of detection are not summarized. Another example of combining methods to achieve better results is presented in [75]. This method is proposed for harmonics estimation in power systems, and shows good results in online tracking of dynamic changes that can be very useful for voltage disturbance analysis. The method combines Least Square (LS) with ADALINE algorithm, to decompose analysis into a linear and a nonlinear part. It shows better performance in comparison with EKF method for tracking harmonics in normal and noisy conditions. Method is not tested for detection of PQ disturbances, but can be very useful for PQ analysis.

Methods that are based on different use of well-known methods are presented in $[76,77]$. Most of the VDDaA methods that are based on WT apply detail coefficient of the highest frequency band for disturbance detection. In [77] method based on improved WT is proposed. This method utilizes two different mother wavelets ( $\mathrm{db} 2$ and $\mathrm{db} 8$ ). Comparison of proposed method with EPLL and FFT is presented. Very good detection times are obtained. However, despite hybrid structure, high frequency noise can deteriorate abilities of proposed algorithm.

ADALINE is an adaptive filter that can be used in extracting signals from noisy environments, in model identification and in linearization of nonlinear problems [78]. In ADALINE is used with AI methods for VDDaA [78], as part of control algorithm of Shunt active power filter [79] and for dynamic phasor estimation [80] and promising results are obtained. In [70] comparison of RMS, ADALINE, AR, ARMA, HPF and WT are presented. ADALINE and RMS detection do not have required precision. WT is suitable for detection of PQ events and reduction of noise enchased performances. However, much higher complexity of AR and ARMA is not justified with only slight improvements in results.

WT is combined with KF to achieve better performances in [81]. Fuzzy-expert system (FES) is used only for classification. Accuracy over $90 \%$ is achieved. The method has the ability to detect and successfully classify different disturbances with relatively low computational complexity. Method that overcomes some known problems of the KF, extended KF (EKF) is applied for detection and classification of voltage disturbances in [82]. EKF method showed good accuracy, but requires all input data for modelling to be known. In [83] hybrid method that includes EKF and ST for detection and analysis of short duration disturbances is addressed. Based on simulation and laboratory research, it is concluded that ST alone can detect and localize disturbances, while KF can successfully extract important parameters of fault. Combined, these two methods show good results in both detection and analysis of disturbances. In [84] the design principles of EKF are presented, together with experimental results and implementation. Based on experimental results of extracting voltage disturbance parameters during transient, it is concluded that estimation includes error and that distortion is present in extracted signal. It is stated that cost of implementation is high because algorithm is highly iterative and needs a fast microprocessor for calculation. However, today's micro-processors can support calculation of EKF with ease. 


\section{Comparative Study}

\subsection{Comparison-based on Overview of Research Papers}

Comparative study of VDDaA is carried out on the basis of critical overview of a large number of findings and conclusions presented in previously published papers. The results of comparative study are presented in Tables 1 and 2. Ten different and the most frequently applied DSP methods have been taken into consideration: RMS, s-RMS, FFT, WT, KF, STFT, ST, HHT and EKF. The methods are commented and rated according to three here defined criteria: 1 . Speed of detection (SoD), 2. Analysis capability (AnC), and 3. Computational demands/cost of implementation (CDi). SoDis time delay between occurrence of disturbance in the grid and its successful detection with tested method. AnC examines method's potential to precisely extract and calculate parameters of a fault that are needed for successful characterization or classification of a VD, and to successfully detect disturbance. CDi is the parameter of a method that defined its complexity, i.e.it can be considered an amount of microprocessor power (time) that must be reserved for implementation of some method, in some hardware unit (e.g. grid-tie inverter control unit or PQ monitoring device).

The SoD, AnC and CDi are rated with numbers from 1 to 10 , where 1 is the worse and 10 is the best, based on results that are presented in literature. As an averaged value, a parameter named averaged Total result $\left(\mathrm{TR}_{\mathrm{a}}\right)$ is introduced and defined with (13). Further on, the three presented criteria are weighted, according to their importance and presented as another new parameter, the weighted Total result $\left(\mathrm{TR}_{\mathrm{w}}\right)$. In this paper, the SoD and $\mathrm{AnC}$ are weighted with coefficients of 0.4 , while CDi is weighted with 0.2 , like it is shown in (14).

$T R_{a}=(S o D+A n C+C D i) / 3$

$T R_{w}=0.4 *(S o D+A n C)+0.2 * C D i$

Table 1 shows advantages and disadvantages of all addressed methods according to the reports in available literature. The methods are not rated. Table 2 presents results of comparison of above mentioned methods according to three here defined criteria and averaged and weighted TR are given.

It is important to notice that some researchers use hybrid methods, which typically contain several DSP methods in combination, while others separately address and test each of them. From these results useful information may be obtained, both about each DSP method and of a whole hybrid algorithm.

The Table 2 shows that WT and HHT methods in the most cases achieve the best overall result. Methods that utilized EKF and ST and STFT follow them as the second best. After these three groups, other popular DSP methods are ranked from place 4 to 10. Standard DSP methods, based on RMS, are ranked with the lowest overall result. 
Table 1

Advantages and disadvantages of DSP methods

\begin{tabular}{|l|l|l|}
\hline & Advantages & Disadvantages \\
\hline $\begin{array}{l}\text { RMS } \\
{[36][37][63]}\end{array}$ & Very simple, standard solution. & $\begin{array}{l}\text { Underperforms in comparison with any } \\
\text { other method. }\end{array}$ \\
\hline $\begin{array}{l}\text { s-RMS } \\
{[36][26]}\end{array}$ & Improved version of RMS. & $\begin{array}{l}\text { Better results than RMS, overall } \\
\text { underperforms. }\end{array}$ \\
\hline $\begin{array}{l}\text { FFT } \\
{[37][50][51]} \\
{[63]}\end{array}$ & $\begin{array}{l}\text { Well known. Standard solution for } \\
\text { harmonics analysis. }\end{array}$ & $\begin{array}{l}\text { Have problems with analysis of } \\
\text { transients. }\end{array}$ \\
\hline $\begin{array}{l}\text { WT } \\
{[37][61][47,} \\
48][53-57]\end{array}$ & Very fast SoD. Better for analysis of \\
\hline $\begin{array}{l}\text { KF } \\
{[63][90][72]}\end{array}$ & $\begin{array}{l}\text { Good amplitude and frequency } \\
\text { estimation capability even in noisy } \\
\text { condition, acceptable SoD and AnC. }\end{array}$ & $\begin{array}{l}\text { Low reliability, prone to noises. Noise } \\
\text { (harmonics) in signal can deteriorate } \\
\text { performances significantly. }\end{array}$ \\
\hline $\begin{array}{l}\text { STFT } \\
{[51][52][61]} \\
{[62]}\end{array}$ & $\begin{array}{l}\text { Good harmonics estimation, useful for } \\
\text { voltage disturbance analysis (better } \\
\text { than WT), good detection abilities. }\end{array}$ & $\begin{array}{l}\text { Induces a significant delay in detection. } \\
\text { Limited performance for analysis of short } \\
\text { duration disturbances. }\end{array}$ \\
\hline $\begin{array}{l}\text { ST } \\
{[30][58][59]} \\
{[83][91]}\end{array}$ & $\begin{array}{l}\text { Works better in noisy conditions than } \\
\text { other FT based methods. }\end{array}$ & $\begin{array}{l}\text { Results in real-time environment are not } \\
\text { good. Because it is based on WT, due to } \\
\text { harmonics estimation has error. }\end{array}$ \\
\hline $\begin{array}{l}\text { HHT } \\
{[49][65][66]} \\
{[92]}\end{array}$ & $\begin{array}{l}\text { Good results in noisy conditions, very } \\
\text { good AC. Good time-frequency } \\
\text { estimation. More adaptive that WT. } \\
\text { Low sensitivity to noise. }\end{array}$ & $\begin{array}{l}\text { Short disturbances transients are difficult } \\
\text { to detect and analyse with HHT. Should } \\
\text { be further tested with real grid } \\
\text { disturbances. }\end{array}$ \\
\hline $\begin{array}{l}\text { EKF } \\
{[37][82][83]} \\
{[84]}\end{array}$ & $\begin{array}{l}\text { Simple, fast SoD. Shows good results } \\
\text { both in detection and analysis. }\end{array}$ & $\begin{array}{l}\text { Results for SoD and AnC are good, but } \\
\text { for AC much better solutions are } \\
\text { proposed. Also, WT have faster SoD. }\end{array}$ \\
& & \\
\end{tabular}

Table 2

Comparison of DSP methods from literature

\begin{tabular}{|l|l|l|l|l|l|l|l|l|l|}
\hline & RMS & s-RMS & FFT & WT & KF & STFT & ST & HHT & EKF \\
\hline SoD (1-10) & 2 & 3 & 4 & 10 & 7 & 7 & 8 & 8 & 8 \\
\hline AnC (1-10) & 2 & 2 & 4 & 5 & 5 & 6 & 6 & 7 & 6 \\
\hline CDi (1-10) & 10 & 9 & 8 & 5 & 7 & 7 & 5 & 5 & 6 \\
\hline TRa (1-10) & 4.67 & 4.7 & 5.33 & 6.67 & 6.4 & 6.67 & 6.3 & 6.67 & 6.67 \\
\hline TRw(1-10) & $\mathbf{3 . 6}$ & $\mathbf{3 . 8}$ & $\mathbf{4 . 8}$ & $\mathbf{7}$ & $\mathbf{6 . 2}$ & $\mathbf{6 . 6}$ & $\mathbf{6 . 6}$ & $\mathbf{7}$ & $\mathbf{6 . 8}$
\end{tabular}

\subsection{Comparison based on Real Grid Measurements and Laboratory Evaluation}

Based on authors previous research [12, 13, 50, 64, 85-88], comprehensive testing with real grid measurements and with grid-emulator in laboratory were done in order to further evaluate presented methods in the same conditions. Out of 680 recordings in real grids, 127 contain some type of voltage dips or interruptions or other disturbances. From these 127 faults, 10 were selected for testing. In selected sample of 10 faults, various types of dips and interruptions are present. Some of them are very interesting, like multiple disturbances and multi-level faults with developing and changing types. 
Rating using AnC criteria is based on ability to detect all disturbances in multipleevents (ME), and on ability to extract key features from all disturbances. Estimated key features must enable proper recognition and classification of each stage of ME. The MOV is magnitude of voltage, which represents minimum value of voltage RMS (calculated with RMS $1 / 2$-cycle) during disturbance, according to [34]. Results of detection time and AnC grade for ten recorded signals of voltage disturbance (dips) based on real grid measurements are presented in Table 3.

The RMS and the s-RMS can obtain only single-event characteristics (duration and magnitude). Because of that, in terms of analyzing they are usually graded with 4 (AnC). The RMS based methods successfully detect start and the end (if it is recorded) of every disturbance, and obtain magnitude. With average detection time of $19.05 \mathrm{~ms}$ and median of $16.7 \mathrm{~ms}$, the RMS 1/2-cycle is the slowest. Estimated magnitude contains less variation in comparison to magnitudes obtained with other methods (s-RMS, FFT, KF and EKF). The s-RMS with average detection time of $12.51 \mathrm{~ms}$ and median of only $7.15 \mathrm{~ms}$ is much better and it does not lag considerably in comparison to more complex methods.

The FFT successfully detected all tested events, and obtained enough information from voltage signal from the most of disturbances, so multiple events can be successful classified. Some information are not extracted precisely, like phase angle in some cases. With average detection time of $11.77 \mathrm{~ms}$, median of $6.31 \mathrm{~ms}$ and considerably good feature extraction, the FFT presents a method that is in the middle of the list by performance. The AnC grade is 7 and reliability is $100 \%$.

The WT detected six out of ten tested faults with reliability of $70 \%$. Such result may be explained by speed of voltage dip amplitude change. The WT cannot detect slowly developing disturbances that have low transient changes despite that signal has low noise level and even using energy of wavelets. But, for more severe disturbances, the WT performs remarkably well, with average detection time of only $4.22 \mathrm{~ms}$ and median of $4 \mathrm{~ms}$, which makes it the fastest method. Also, the WT enables successful classification of a disturbance, even if it is complex one. Because of low reliability AnC grade is 6, but SoD grade is 10 .

The KF and EKF are applied in a similar way, using fundamental harmonic for a model. The EKF is more complex and better in dynamic state estimation, as it is modified version of linear KF. The EKF's average detection time is $8.08 \mathrm{~ms}$ with median of $4.76 \mathrm{~ms}$. Only for one shallow dip, the EKF underperform with 28.1 $\mathrm{ms}$. The KF average detection time is $11.74 \mathrm{~ms}$ with median $6.1 \mathrm{~ms}$. Both methods have reliability of $100 \%$, with $\mathrm{AnC}$ grade of 7.8.SoD grades are 6 and 8 for $\mathrm{KF}$ and EKF, respectively.

All voltage dips detection methods are tested in laboratory conditions, as well, using voltage dips which have been generated by a grid-emulator. The detection methods were applied as part of grid-tie inverter control. Primary task was to observe the methods' behaviour in real-time systems, measure computational complexity in real-time environment, and compare methods from viewpoint of 
ease-of-use. Fig. 3 presents overall look of such laboratory setup. It consists of advanced hardware in the field of electrical drives and of the control units based on highly modular dSpace control hardware and modified industrial converters [89]. The system is paired with AC grid emulator GE 15-AC and connected using Yd transformer to the supply. Computational complexity is measured on dSpace, which utilizes DS1006 processor board (AMD Opteron ${ }^{\mathrm{TM}}$ processor). System is set to works at a PWM frequency of $6.4 \mathrm{kHz}$ and generates a synchronized software interrupt with a $3.2 \mathrm{kHz}$ frequency.

Table 3

Real grid method testing - detection times, reliability and analysis capability

\begin{tabular}{|c|c|c|c|c|c|c|c|}
\hline \multirow[b]{2}{*}{$\begin{array}{l}\text { Description of } \\
\text { disturbances / No. }\end{array}$} & \multirow{2}{*}{$\begin{array}{c}\text { MOV } \\
{[\%]}\end{array}$} & \multicolumn{6}{|c|}{ Detection time [ms] / AnC [1-10] } \\
\hline & & RMS & $\begin{array}{c}\text { S- } \\
\text { RMS }\end{array}$ & FFT & WT & $\mathrm{KF}$ & EKF \\
\hline $\begin{array}{l}\text { \#1. Type G, five cycles, } \\
\text { develops into Type A }\end{array}$ & 79 & $22.6 / 4$ & $19.7 / 4$ & $19 / 7$ & $4.4^{\mathrm{e}} / 8$ & $19.5 / 7$ & $10.3 / 7$ \\
\hline$\# 2$. Non-fault interruption & 5.5 & $35.3 / 4$ & $6.29 / 5$ & $4.6 / 10$ & $7.34 / 10$ & $4.4 / 10$ & $3.77 / 10$ \\
\hline \#3. Type C, 15 cycles & 87 & $39.7 / 4$ & $40.4 / 4$ & $39 / 7$ & I & $39.9 / 7$ & $28.1 / 7$ \\
\hline $\begin{array}{l}\text { \#4. Balanced dip with } \\
\text { unbalanced recovery }\end{array}$ & 48 & $9.5 / 5$ & $7.35 / 5$ & $6.3 / 8$ & $3.5^{\mathrm{e}} / 9$ & $6.1 / 8$ & $4.1 / 8$ \\
\hline $\begin{array}{l}\text { \#5. Remarkable multiple } \\
\text { event * }\end{array}$ & 59 & $7.2 / 4$ & $6.6 / 4$ & $5.9 / 8$ & $3^{\mathrm{e} 1} / 9$ & $5.7 / 8$ & $3.6 / 8$ \\
\hline \#6. Type D dip & 56 & $9.19 / 4$ & $4.15 / 4$ & $3.7 / 8$ & I & $3.72 / 8$ & $3.26 / 8$ \\
\hline \#7. Type F, 15 into type A & 84 & $28.7 / 4$ & $21.8 / 4$ & $21 / 8$ & l & $21.5 / 8$ & $13.4 / 8$ \\
\hline \#8. Three-phase fault & 47 & $16.2 / 4$ & $6.95 / 4$ & $6.3 / 8$ & $3.1^{\mathrm{e} 1} / 10$ & $6.11 / 8$ & $5.7 / 8$ \\
\hline $\begin{array}{l}\text { \#9. Unbalanced dip (Type } \\
\text { C). }\end{array}$ & 67 & $4.9 / 4$ & $4.3 / 4$ & $3.6 / 9$ & $4 / 8$ & $3.6 / 9$ & $3.2 / 9$ \\
\hline $\begin{array}{l}\text { \#10. Single-phase fault } \\
\text { with over-voltages } \\
\text { (multiple events) }\end{array}$ & 0.47 & $17.2 / 5$ & $7.53 / 5$ & $6.91 / 8$ & $4.17^{\mathrm{e} 1} / 9$ & $6.9 / 8$ & $5.43 / 8$ \\
\hline Average detection time & & 19.05 & 12.51 & 11.77 & 4.22 & 11.74 & 8.08 \\
\hline Reliability [\%] & & 100 & 100 & 100 & $\mathbf{7 0}$ & 100 & 100 \\
\hline Mean of AnC [1-10] & & 4.2 & 4.3 & 7 & 6 & 7.8 & 7.8 \\
\hline
\end{tabular}

${ }^{\mathrm{e}}$ Energy of wavelet is used for detection, fault cannot be detected with detail coefficients; ${ }^{\mathrm{el}}$ Energy of wavelet is used for detection, but fault can be detected with detail coefficient/detection is slower; *Starts as type C, slow recovery one phase up, two phases down, repeat of the first event

The testing showed that all methods were successfully applied. Results of digital processor computation times are presented in Table 4. Standard RMS method is one with the lowest execution time, following with the s-RMS, FFT, KF, EKF and WT, with delays of $8 \%, 57 \%, 61 \%, 71 \%$, and $146 \%$, respectively.

In Fig. 4 graphical presentation of DSP VDDaA method results are shown. In Fig. 5 examples of signal processing with 3 tested methods are given. Signal of disturbance fault \#10 from Table 3 is analysed with different algorithms: RMS, FFT and WT. Outputs of these algorithms are presented. Fig. 5a presents voltage signals recorded in real grid, Fig. 5 b shows time representation obtained with the 
RMS, Fig. 5c shows frequency representation obtained with the FFT and Fig. 5d shows time-frequency representation derived using the WT.

Based on SoD, AnC and CDi presented in Tables 3 and 4, a comparison of all tested methods is given in Table 5. Also, $\mathrm{TR}_{\mathrm{a}}$ and $\mathrm{TR}_{\mathrm{w}}$ are calculated. Ratings for SoD were presented in a way that the best method obtained rating 10 , as shorter time presents better result. It can be seen that the WT based method that analyzes voltage signals is the best in term of detection speed, but has lower CDi and reliability problems.EKF shows the best overall results of 7.72, while WT, KF and FFT follows (7.4, 7.12 and 6.8 respectively). RMS based methods underperforms.

\section{Conclusion}

A comprehensive and critical review on methods for detection and analysis of voltage disturbances, based on DSP methods is presented. The major advantages and disadvantages are outlined, as well as the comparison of the wide range of methods in the DSP domain.

Based on comprehensive laboratory and real grid measurement signals testing, it can be concluded that EKF and WT have the best overall grades. Also, the FFT and KF can be distinguished as the ones with high detection capabilities. On the other hand, the RMS based method underperforms. However, it should be noted that each of these methods has its own advantages and drawbacks, and selection should be done based on specific application and priorities. Based on presented comprehensive literature review, it can be concluded that the DSP techniques can be successfully used for VDDaA in modern distribution grids.

Signals with significant amount of noise are challenge even for advanced methods, and detection and analysis methods can underperform due to noise in signals. Also, complex multiple disturbances, or very distant disturbances that cause shallow dips may be challenging, as well.

Table 4

Microprocessor execution time of a voltage detection method

\begin{tabular}{|l|c|c|c|c|c|c|}
\hline & RMS & s-RMS & FFT & WT & KF & EKF \\
\hline $\begin{array}{l}\text { Laboratory execution } \\
\text { time on dSpace }[\mu \mathrm{s}]\end{array}$ & 7.1 & 7.67 & 11.2 & 17.5 & 11.5 & 12.2 \\
\hline
\end{tabular}

Table 5

Comparison of DSP methods based on comprehensive evaluation

\begin{tabular}{|l|l|l|l|l|l|l|}
\hline & RMS & s-RMS & FFT & WT & KF & EKF \\
\hline SoD (1-10) & 3 & 6 & 6 & 10 & 6 & 8 \\
\hline AnC (1-10) & 4.2 & 4.3 & 7 & 6 & 7.8 & 7.8 \\
\hline CDi (1-10) & 10 & 10 & 8 & 5 & 8 & 7 \\
\hline TR $_{\mathbf{a}}(\mathbf{1 - 1 0})$ & $\mathbf{5 . 7 3}$ & $\mathbf{6 . 7 7}$ & $\mathbf{7}$ & $\mathbf{7}$ & $\mathbf{7 . 2 7}$ & $\mathbf{7 . 6}$ \\
\hline TR $_{\mathbf{w}}(\mathbf{1 - 1 0})$ & $\mathbf{4 . 8 8}$ & $\mathbf{6 . 1 2}$ & $\mathbf{6 . 8}$ & $\mathbf{7 . 4}$ & $\mathbf{7 . 1 2}$ & $\mathbf{7 . 7 2}$ \\
\hline
\end{tabular}




\section{Future scope}

Despite large amount research results in the field of VDDaA, several challenges remain. There is still space to find better method in terms of the detection and analysis performances, and to optimize it in term of computational complexity using artificial intelligence techniques. Also, improvements and additional research in finding a method that has the ability to provide good results in noisy conditions and in analysing events with multiple disturbances are needed.

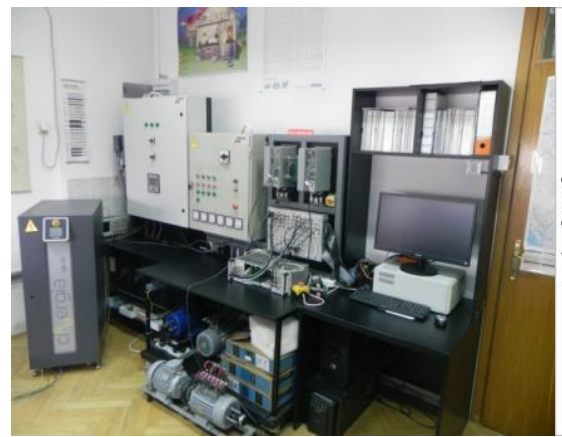

Fig. 3 Outlook of the laboratory setup.
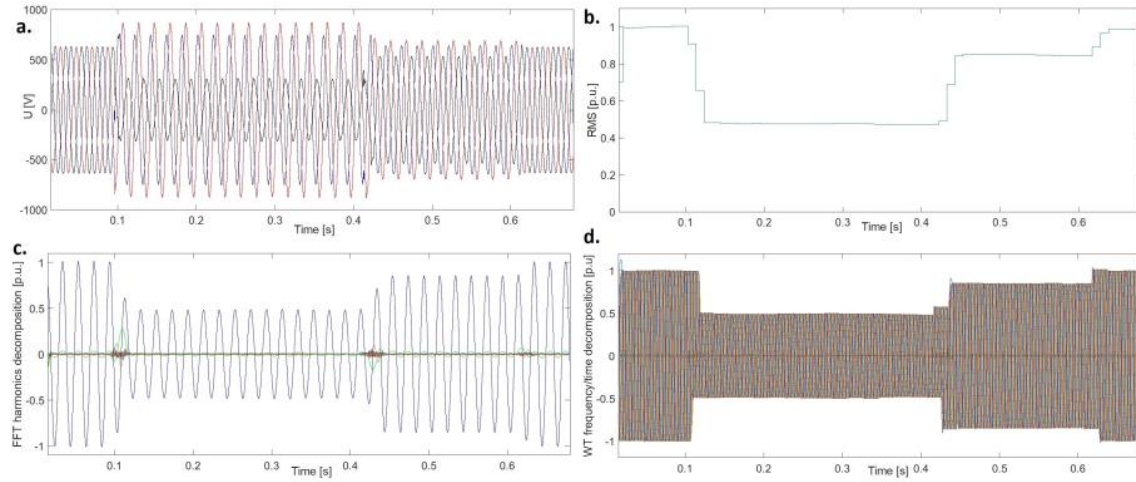

Fig. 5a Voltage signal for fault \#10 (Table 3) processing with: b. RMS, c. FFT harmonics decomposition, d. WT frequency/time decomposition

\section{Acknowledgement}

This work was supported by the Republic of Serbia, Ministry of Education, Science and Technological Development, project No. III 042004 entitled "Smart Electricity Distribution Grids Based on Distribution Management System and Distributed Generation“.

The authors would like to express their acknowledgement to Prof. Math Bollen who has provided in-field measurement results. 


\section{References}

[1] Bollen, M. H.: Understanding Power Quality Problems, IEEE, 1999

[2] Bae, Y. et al.: Implemental control strategy for grid stabilization of gridconnected PV system based on German grid code in symmetrical low-tomedium voltage network, IEEE Tran. En. Conv., 28(3), 2013, pp. 619-631

[3] Yang, Y. et al.: Benchmarking of Grid Fault Modes in Single-Phase GridConnected Photovoltaic Systems, IEEE Trans. Ind. Appl., 49 (5), 2013, pp. 2167-2176

[4] Yang, Y., Blaabjerg, F.: Low-voltage ride-through capability of a singlestage single-phase photovoltaic system connected to the low-voltage grid, Int. J. Photoenergy, 2013, pp. 1-9

[5] Bhavaraju, V. B., Enjeti, P.: A Fast Active Power Filter to Correct Line Voltage Sags, IEEE Trans. Ind. Electron., 41 (3), 1994, pp. 333-338

[6] Akagi, H.: New trends in active filters for power conditioning, IEEE Trans. Ind. Appl., 32 (6), 1996, pp. 1312-1322

[7] Nielsen, J. G., Blaabjerg, F.: A detailed comparison of system topologies for dynamic voltage restorers, IEEE Tran. Ind. Appl., 41(5), 2005, pp. $1272-1280$

[8] Rufer, A. et al.: Power quality compensation using universal power quality conditioning system, IEEE Power Eng. Rev., 20 (12), 2000, pp. 58-60

[9] Kwan, K. H. et al.: An output regulation-based unified power quality conditioner with Kalman filters, IEEE Trans. Ind. Electron., 59 (11), 2012, pp. $4248-4262$

[10] Zhao, W. et al.: Microgrid relay protection scheme based on harmonic footprint of short-circuit fault, Chinese J. Elec. Eng., 4(4), 2018, pp. 64-70

[11] Afshari, E. et al.: Control Strategy for Three-Phase Grid-Connected PV Inverters Enabling Current Limitation under Unbalanced Faults, IEEE Trans. Ind. Electron., 64 (11), 2017, pp. 8908-8918

[12] Katic, V. A., Stanisavljevic, A. M.: Smart Detection of Voltage Dips Using Voltage Harmonics Footprint, IEEE Trans. Ind. Appl., 54 (5), 2018, pp. $5331-5342$

[13] Katic, V. A., Stanisavljevic, A. M.: Novel voltage dip detection algorithm using harmonics in the dip's transient stage, Proc. IECON 2017 - 43rd Annu. Conf. IEEE Ind. Electron. Soc., 2017, pp. 351-356

[14] Granados-Lieberman, D. et al.: Techniques and methodologies for power quality analysis and disturbances classification in power systems: a review, IET Gener. Transm. Distrib., 5 (4), 2011, p. 519 
[15] Abdelsalam, A. A. et al.: Classification of power system disturbances using linear Kalman filter and fuzzy-expert system, Int. J. Electr. Power Energy Syst., 43 (1), 2012, pp. 688-695

[16] Borges, F. A. S. et al.: Feature Extraction and Power Quality Disturbances Classification Using Smart Meters Signals, IEEE Trans. Ind. Informatics, 12 (2), 2016, pp. 824-833

[17] Blackledge, J.: Digital Signal Processing ( ${ }^{\text {nd }}$ Ed.), Horwood Publ., 2006

[18] Antoniou, A.: Digital Signal Processing: Signals, Systems and Filters, McGraw-Hill, 2016

[19] Haidegger, T. et al.: Simulation and control for telerobots in space medicine, Acta Astronaut., 81 (1), 2012, pp. 390-402

[20] Spall, J. C.: Multivariate Stochastic Approximation Using a Simultaneous Perturbation Gradient Approximation, IEEE Trans. Automat. Contr., 37 (3), 1992, pp. 332-341

[21] Ürmös, A. et al.: Application of Self-Organizing Maps for Technological Support of Droplet Epitaxy, Acta Polytechnica Hungarica, 14 (4), 2017, pp. 207-224

[22] Khokhar, S. et al.: A comprehensive overview on signal processing and artificial intelligence techniques applications in classification of power quality disturbances, Renew. Sust. Energy Rev., 51, 2015, pp. 1650-1663

[23] Hosseini, S. A. et al.: An overview of microgrid protection methods and the factors involved, Renew. Sust. Energy Rev., 64, 2016, pp. 174-186

[24] Saini, M. K., Kapoor, R.: Classification of power quality events - A review, Int. J. Electr. Power Energy Syst., 43 (1), 2012, pp. 11-19

[25] Mahela, O. P. et al.: A critical review of detection and classification of power quality events, Renew. Sust. Energy Rev., 41, 2015, pp. 495-505

[26] Gururajapathy, S. S. et al.: Fault location and detection techniques in power distribution systems with distributed generation: A review, Renew. Sust. Energy Rev., 74, 2017, pp. 949-958

[27] Khokhar, S. et al.: Automatic Classification of Power Quality Disturbances : A Review, IEEE Student Conf. Res. Dev., 2013, pp. 16-17

[28] Barros, J. et al.: Review of signal processing techniques for detection of transient disturbances in voltage supply systems, IEEE Instrum. Meas. Technol. Conf., 2013, pp. 450-455

[29] Prakash M. O., Gafoor S. A.: Topological aspects of power quality improvement techniques: A comprehensive overview, Renew. Sust. Energy Rev., 58, 2016, pp. 1129-1142 
[30] Mishra, S. et al.: Detection and Classification of Power Quality Disturbances Using S-Transform and Probabilistic Neural Network, IEEE Trans. Power Deliv., 23 (1), 2008, pp. 280-287

[31] Hooshmand, R., Enshaee, A.: Detection and classification of single and combined power quality disturbances using fuzzy systems oriented by particle swarm optimization algorithm, Electr. Power Syst. Res., 80 (12), 2010, pp. 1552-1561

[32] Bollen, M. H. J., Gu, I. Y. H.: Signal processing of power quality disturbances, New York: Press, Series on Power Eng., 2006

[33] Institute of Electrical and Electronics Engineers: 1159-2009 - IEEE Recommended Practice for Monitoring Electric Power Quality, 2009

[34] Institute of Electrical and Electronics Engineers: IEEE Std 1564 - Guide for Voltage Sag Indices, 2014

[35] International Electrotechnical Commission: Electromagnetic compatibility (EMC): IEC 61000-4-30 Edition 3.0 2015-02, 2015

[36] Albu, M., Heydt, G. T.: On the use of RMS values in power quality assessment, IEEE Trans. Power Deliv., 18 (4), 2003, pp. 1586-1587

[37] Perez, E., Barros, J.: Voltage Event Detection and Characterization Methods: A Comparative Study, IEEE/PES Transm. Distrib. Conf. Exp. Lat. Am., 2006, pp. 1-6

[38] Jain, S. K., Singh, S. N.: Harmonics estimation in emerging power system: Key issues and challenges, Electr. Power Syst. Res., 81 (9), 2011, pp. 17541766

[39] Huang, N. E. et al.: The Empirical Mode Decomposition and the Hilbert Spectrum for Nonlinear and Non- Stationary Time Series Analysis, Proc. R. Soc. London A, 454 (1971), 1998, pp. 903-995

[40] Zhao, F., Yang, R.: Power-quality disturbance recognition using Stransform, IEEE Trans. Power Deliv., 22 (2), 2007, pp. 944-950

[41] Aiello, M. et al.: Synchronization techniques for power quality instruments. IEEE Trans. Instrum. Meas., 56 (5), 2007, pp. 1511-1519

[42] Belega, D., Petri, D.: Frequency estimation by two- or three-point interpolated Fourier algorithms based on cosine windows, Signal Processing, 117, 2015, pp. 115-125

[43] Chintakindi, S. R. et al.: Improved Hanning window based interpolated FFT for power harmonic analysis, IEEE TENCON, 2016, pp. 1-5

[44] Wen, H. et al.: Harmonic Estimation Using Symmetrical Interpolation FFT Based on Triangular Self-Convolution Window, IEEE Trans. Ind. Informatics, 11 (1), 2015, pp. 16-26 
[45] Belega, D. et al.: Iterative sine-wave frequency estimation by generalized Fourier interpolation algorithms, $11^{\text {th }}$ Int. Symp. Electron. Telecommun. ISETC 2014 - Conf. Proc., 2014, pp. 1-4

[46] Van Der Byl, A., Inggs, M. R.: Recursive sliding discrete Fourier transform with oversampled data, Digit. Sig. Proc. A Rev. J., 25(1), 2014, pp. 275279

[47] Thirumala, K. et al.: Estimation of single-phase and three-phase powerquality indices using empirical wavelet transform, IEEE Trans. Power Deliv., 30 (1), 2015, pp. 445-454

[48] Poisson, O. et al.: Detection and measurement of power quality disturbances using wavelet transform, IEEE Trans. Power Deliv., 15 (3), 2000, pp. 1039-1044

[49] Yang, L. et al.: Disturbance source identification of voltage sags based on Hilbert-Huang transform, APPEEC, 2010, pp.1-4

[50] Stanisavljevic, A. M. et al.: Voltage dips detection in a system with grid-tie inverter, Proc. of $18^{\text {th }}$ EPE 2016 ECCE Europe, 2016, pp. 1-10

[51] Ingale, R.: Harmonic Analysis Using FFT and STFT, Int. J. Signal Process. Image Process. Pattern Recognit., 7 (4), 2014, pp. 345-362

[52] Jurado, F., Saenz, J. R.: Comparison between discrete STFT and wavelets for the analysis of power quality events, Electr. Power Syst. Res., 62 (3), 2002, pp. 183-190

[53] Santoso, S. et al.: Power quality assessment via wavelet transform analysis, IEEE Trans. Power Deliv., 11 (2), 1996, pp. 924-930

[54] Lin, C.-H., Tsao, M.-C.: Power quality detection with classification enhancible wavelet-probabilistic network in a power system, IEE Proc. Gener. Transm. Distrib., 152 (6), 2005, pp. 969-976

[55] Barros, J. et al.: Applications of wavelets in electric power quality: Voltage events, Electr. Power Syst. Res., 88, 2012, pp. 130-136

[56] Kezunovic, M., Liao, Y.: A novel software implementation concept for power quality study, IEEE Trans. Power Deliv., 17 (2), 2002, pp. 544-549

[57] Perez, E., Barros, J.: A proposal for on-line detection and classification of voltage events in power systems, IEEE Trans. Power Deliv., 23 (4), 2008, pp. 2132-2138

[58] He, S. et al.: A real-time power quality disturbances classification using hybrid method based on s-transform and dynamics, IEEE Trans. Instrum. Meas., 62 (9), 2013, pp. 2465-2475

[59] Ray, P. K. et al.: Islanding and Power Quality Disturbance Detection in Grid-Connected Hybrid Power System Using Wavelet and S-Transform, IEEE Trans. Smart Grid, 3 (3), 2012, pp. 1082-1094 
[60] Dash, P. et al.: Power quality analysis using s-transform, IEEE Trans. Power Deliv., 18 (2), 2003, pp. 406-411

[61] Gu, Y., Bollen, M. H. J.: Time-frequency and time-scale domain analysis of voltage disturbances, IEEE Tran. Power Deliv., 15(4), 2000, pp.1279-1284

[62] Ingale, R., Tawade, L.: Detection and Comparison of Power Quality Disturbances using Different Techniques, Int. J. Comput. Appl., 75 (18), 2013, pp. 48-53

[63] Amarís, H. et al.: Computation of voltage sag initiation with Fourier based algorithm, Kalman filter and Wavelets, 2009 IEEE Bucharest PowerTechInnov. Ideas TowardElectr. Grid Futur., 2009, pp. 1-6

[64] Stanisavljevic, A. M. et al.: Wavelet transform for voltage dips detection in a microgrid with distributed generation, Proc. of $19^{\text {th }}$ EPE'17 ECCE Europe, 2017, pp. 1-10

[65] Li, Y. et al.: Study on Voltage Sag Detection of Wind Power System Based on HHT, Energy and Power Engineering, 05 (04), 2013, pp. 922-926

[66] Afroni, M. J. et al.: Analysis of nonstationary power-quality waveforms using iterative Hilbert Huang transform and sax algorithm, IEEE Trans. Power Deliv., 28 (4), 2013, pp. 2134-2144

[67] Das, D. et al.: Hilbert huang transform with fuzzy rules for feature selection and classification of power quality disturbances, Proc. of $4^{\text {th }}$ IEEE Uttar Pradesh Sect. Int. Conf. Electr. Comput. Electron., 2017, pp. 439-445

[68] Gu, I. Y. H. et al.: The use of time-varying AR models for the characterization of voltage disturbances, Proc. of IEEE Power Eng. Soc. Conf., 2000, pp. 2943-2948

[69] Li, S., Wang, X.: Cooperative Change Detection for Voltage Quality Monitoring in Smart Grids, IEEE Trans. Inf. Forensics Secur., 11 (1), 2016, pp. 86-99

[70] Chang, G. W., Cheng-I Chen: Performance evaluation of voltage sag detection methods, Proc. of IEEE PES General Meeting, 2010, pp. 1-6

[71] Moreno Saiz, V. M., Barros Guadalupe, J.: Application of Kalman filtering for continuous real-time tracking of power system harmonics, IEE Proc. Gener. Transm. Distrib., 144 (1), 1997, p. 13

[72] Barros, J. Perez, E.: Automatic Detection and Analysis of Voltage Events in Power Systems, IEEE Trans. Inst. Meas., 55 (5), 2006, pp. 1487-1493

[73] Stanisavljevic, A. M. et al.: Overview of voltage dips detection analysis methods. Proc. of $19^{\text {th }}$ Intern. Symposium on Power Elec., 2017, pp. 1-6

[74] De Apráiz, M. et al.: A real-time method for time-frequency detection of transient disturbances in voltage supply systems, Electr. Power Syst. Res., 108, 2014, pp. 103-112 
[75] Joorabian, M. et al.: Harmonic estimation in a power system using a novel hybrid Least Squares-Adaline algorithm. Electr. Power Syst. Res., 79 (1), 2009, pp. 107-116

[76] Costa, F. B. et al.: Assessment of Voltage Sag Indices Based on Scaling and Wavelet Coef fi cient Energy Analysis,IEEE Trans. on Power Delivery, 28 (1), 2013, pp. 336-346

[77] Latran, M. B., Teke, A.: A novel wavelet transform based voltage sag/swell detection algorithm, Int. J. Elec. Power Ener. Syst., 71, 2015, pp.131-139

[78] Valtierra-Rodriguez, M. et al.: Detection and classification of single and combined power quality disturbances using neural networks, IEEE Trans. Ind. Electron., 61 (5), 2014, pp. 2473-2482

[79] Martinek, R. et al.: An Efficient Control Method of Shunt Active Power Filter Using ADALINE, IFAC-PapersOnLine, 49 (25), 2016, pp. 352-357

[80] Nanda, S., Dash, P. K.: A Gauss-Newton ADALINE for dynamic phasor estimation of power signals and its FPGA implementation, IEEE Trans. Instrum. Meas., 67 (1), 2018, pp. 45-56

[81] Abdelsalam, A. A. et al.: Characterization of power quality disturbances using hybrid technique of linear Kalman filter and fuzzy-expert system, Electr. Power Syst. Res., 83 (1), 2012, pp. 41-50

[82] Ghahremani, E., Kamwa, I.: Dynamic state estimation in power system by applying the Extended Kalman filter with unknown inputs to phasor measurements, IEEE Trans. Power Syst., 26 (4), 2011, pp. 2556-2566

[83] Dash, P. K., Chilukuri, M. V.: Hybrid S-transform and Kalman filtering approach for detection and measurement of short duration disturbances in power networks, IEEE Trans.Instrum. Meas., 53 (2), 2004, pp. 588-596

[84] Routray, A. et al.: A novel Kalman filter for frequency estimation of distorted signals in power systems, IEEE Trans. Instrum. Meas., 51 (3), 2002, pp. 469-479

[85] Katic, V. A. et al.: Comparison of voltage dips detection techniques in microgrids with high level of distributed generation, Proc. of $17^{\text {th }}$ IEEE Intern. Conf. on Smart Technologies, EUROCON, 2017, pp. 1-6

[86] Stanisavljević. A. M. et al.: Voltage dips detection using Kalman filter in a microgrid with high level of distributed generation, Proc. of $20^{\text {th }}$ EPE 2018 ECCE Europe, 2018, pp. 1-10

[87] Stanisavljević, A. M. et al.: Reduced FFT algorithm for network voltage disturbances detection, Proc. of Int. Sym. Ind. Elect. INDEL, 2016, pp. 1-6

[88] Stanisavljevic, A. M. et al.: Voltage dips detection in a microgrid with distributed generation for grid-tie inverter protection purposes. Proc. of $19^{\text {th }}$ EPE 2017 ECCE Europe, 2017, pp. 1-10 
[89] Dumnic, B. et al.: Advanced laboratory setup for control of electrical drives as an educational and developmental tool, Proc. of $15^{\text {th }}$ IEEE Intern. Conf. EUROCON, 2013, pp.903-909

[90] Moreno Saiz, V. M., Barros Guadalupe, J.: Application of Kalman filtering for continuous real-time tracking of power system harmonics, IEE Proc. Gener. Transm. Distrib., 144 (1), 1997, pp. 13-21

[91] Mohanty, S. R. et al.: Classification of disturbances in hybrid DG system using modular PNN and SVM, Int. J. Electr. Power Energy Syst., 44 (1), 2013, pp. 764-777

[92] Das, D. et al.: Hilbert Huang transform with fuzzy rules for feature selection and classification of power quality disturbances, Proc. of $4^{\text {th }}$ IEEE Uttar Pradesh Sect. Int. Conf. Electr. Comput. Electron., 2017, pp. 439-445 